03

\title{
Дифференциальные характеристики поля течения перерасширенной газовой струи в окрестности кромки сопла
}

\author{
(C) М.В. Чернышов, ${ }^{1}$ Л.Г. Гвоздева ${ }^{2}$ \\ ${ }^{1}$ Балтийский государственный технический университет «BOEHMEX» им. Д.Ф. Устинова, \\ 190005 Санкт-Петербург, Россия \\ ${ }^{2}$ Объединенный институт высоких температур РАН, \\ 125412 Москва, Россия \\ e-mail: mvcher@mail.ru
}

(Поступило в Редакцию 27 октября 2017 г. В окончательной редакции 25 сентября 2018 г.)

\begin{abstract}
Проведено параметрическое исследование особенностей поля течения плоской и осесимметричной перерасширенной струи идеального газа в окрестности кромки сопла во всем теоретически допустимом диапазоне определяющих параметров (углов раствора сопла, чисел Маха истечения, нерасчетностей струи, показателей адиабаты газа). Выявлены параметры истечения, соответствующие экстремумам дифференциальных характеристик скачка уплотнения, падающего (сходящего) с кромки, и поля течения за ним. Найдено и исследовано значительное различие в характере изменения характеристик скачка и поля течения за ним в зависимости от типа симметрии газовой струи.
\end{abstract}

DOI: 10.21883/JTF.2019.04.47300.2533

\section{Введение}

В настоящей работе проведен теоретический анализ поля течения перерасширенной газовой струи в окрестности кромки сопла. Получены аналитические соотношения, описывающие особые свойства течения: нулевые и экстремальные значения геометрической кривизны границы струи и скачка уплотнения, сходящего с кромки; экстремумы завихренности потока в сжатом слое за скачком и условия смены ее направленности; нулевые и экстремальные значения градиентов полного и статического давлений в сжатом слое; другие особенности поля течения, непосредственно определяемые формой падающего скачка (например, изменение числа Маха за ним). Настоящая работа представляет собой первую попытку максимально полного анализа влияния всех определяющих параметров (нерасчетности струи, числа Маха истечения, угла раствора сопла, показателя адиабаты газа, вида симметрии потока) на особенности поля течения, перечисленные выше.

Несмотря на применение идеализированной математической модели (рассматривается невязкое течение совершенного газа), полученные теоретические результаты выдерживают сравнение с экспериментальными данными $[1,2]$ и могут быть использованы для анализа ряда физических свойств сверхзвуковой струи:

1. Образование неустойчивости Тейлора-Гертлера $[1,3-5]$. Согласно [6-8], геометрическая кривизна границы вместе с поперечным градиентом статического давления в ее окрестности - основные параметры, определяющие возникновение и развитие этой продольной неустойчивости на границе струи.

2. Особенности регулярного и маховского отражений скачка уплотнения, сходящих с кромки $[9,10]$. Значительная часть выявленных особых свойств относится к диапазону параметров истечения, в котором отсутствуют теоретические решения как для регулярного, так и для маховского отражений скачка уплотнения от плоскости или оси симметрии. Связь выявленных особенностей поля течения с дальнейшим отражением скачка подлежит дальнейшему анализу.

3. Автоколебательные режимы взаимодействия струйных течений с преградой [11]. Большие градиенты давления торможения за сходящим с кромки скачком и связанная с ними завихренность поля течения способны привести к возбуждению автоколебательных режимов, что также подлежит дальнейшему исследованию.

Для теоретического анализа использовались дифференциальные условия динамической совместности соотношения между пространственными производными параметров поля течения на сторонах газодинамических разрывов, записанные в $[12,13]$ в форме, наиболее удобной для практического применения. Другие формы записи этих соотношений, применяемые, начиная с 1950х г. [14-18], по нашему мнению, требуют адаптации для решения практически важных задач.

\section{Схема течения. Основные соотношения}

Скачок уплотнения $A T$ (рис. 1), сходящий с кромки сопла $A$ в перерасширенной струе невязкого совершенного газа, истекающей из ракетного сопла, характеризуется своей интенсивностью $[12,19-21] J=p_{n} / p_{a}$, где $p_{n}$ — давление в затопленном пространстве, $p_{a}$ - статическое давление газового потока перед скачком (в устье струи). Обратное отношение давлений $n=p_{a} / p_{n}=1 / J$ называется нерасчетностью (в перерасширенной струе $n<1$ ). 

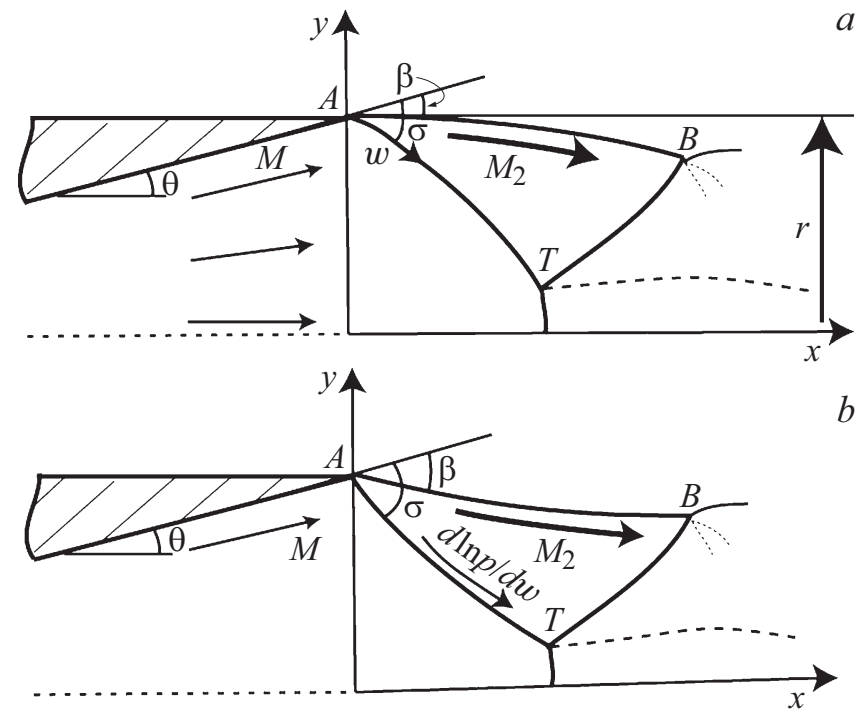

Pис. 1. Схема течения на начальном участке перерасширенной газовой струи: $a-$ традиционная „бочкообразная“ форма начального участка; $b-$ положительная кривизна скачка уплотнения и границы струи.

Интенсивность скачка, существующего в потоке с фиксированным числом Маха $M$ и показателем адиабаты газа $\gamma$, принимает значения из диапазона $1<J \leq J_{m}$, где предел $J \rightarrow 1$ соответствует вырождению скачка в слабое возмущение, а $J_{m}=\left\{(1+\varepsilon) M^{2}-\varepsilon\right\}-$ интенсивность прямого скачка (здесь и далее $\varepsilon=(\gamma-1)(\gamma+1)$, и $\gamma=1.4$ по умолчанию во всех примерах расчета).

Интенсивность скачка уплотнения связана с его формой соотношением

$$
J=(1+\varepsilon) M^{2} \sin ^{2} \sigma-\varepsilon,
$$

где $\sigma$ - угол наклона скачка к линиям тока перед ним.

Угол $\beta$ поворота потока на скачке уплотнения зависит от его интенсивности и числа Маха перед ним следующим образом:

$$
\beta=\operatorname{arctg}\left[\sqrt{\frac{J_{m}-J}{J+\varepsilon}} \frac{(1-\varepsilon)(J-1)}{(1+\varepsilon) M^{2}-(1-\varepsilon)(J-1)}\right] .
$$

Интенсивность

$$
J_{\Gamma}=M^{2}-1
$$

при $M>\sqrt{2}$ соответствует максимальному значению угла поворота потока на множестве всех скачков с фиксированными значениями $J$ и $\gamma$, но переменным числом Маха $M$ перед ними (огибающей семейства ударных поляр).

Дифференциальные характеристики поля двумерного изоэнергетического течения идеального невязкого газа определяются основными неравномерностями $N_{i} \quad(i=1 \ldots 3)$, входящими в уравнения движения $[12,13,20]$, записанные в „естественных“ координа-
$\operatorname{Tax}(s, n)$ :

$$
\begin{gathered}
\frac{M^{2}-1}{\gamma M^{2}}+\frac{\partial \theta}{\partial n}+N_{4} \sin \theta=0, \\
\gamma M^{2} N_{2}=-\partial \ln p / \partial n, \quad \partial p_{0} / \partial s=0 .
\end{gathered}
$$

Величина $N_{1}=\partial \ln p / \partial s$ характеризует неизобаричность течения, $N_{2}=\partial \theta / \partial s$ - геометрическая кривизна линий тока, неравномерность $N_{3}=\partial \ln p_{0} / \partial s$ служит мерой завихренности потока. Здесь $p-$ статическое, $p_{0}$ - полное давление, $\theta-$ угол течения относительно плоскости или оси симметрии, в окрестности кромки, совпадающий с полууглом раствора сопла.

Основные неравномерности поля течения на сторонах скачка связаны дифференциальными условиями динамической совместности $[12,13,20-23]$ :

$$
\tilde{N}_{i}=C_{i} \sum_{j=1}^{5} A_{i j} N_{j}
$$

где $\tilde{N}_{i}(i=1 \ldots 3)$ - неравномерности потока за скачком, $N_{j}(j=1 \ldots 3)$ - перед ним, $N_{4}=\delta / \gamma-$ показатель симметрии ( $\delta=0$ в плоском, $\delta=1$ в осесимметричном течении, $y$ - расстояние от плоскости или оси симметрии), $N_{5} \equiv K_{\sigma}-$ геометрическая кривизна скачка. Коэффициенты $C_{i}$ и $A_{i j}$ определяются [12,13] значениями $M, J$ и $\gamma$, а в осесимметричном потоке также и углом течения $\theta$.

Условие $A_{15}=0$ в (3) определяет единственную на каждой ударной поляре интенсивность $J_{p}$ скачка уплотнения, при которой геометрическая кривизна скачка не оказывает влияния на локальное значение неизобаричности за ним (так называемую точку постоянного давления). Интенсивность скачка уплотнения в этой точке

$$
\begin{aligned}
J_{p}= & {\left[3(1+\varepsilon) M^{2}-2(1+3 \varepsilon)+(1+\varepsilon)\right.} \\
& \left.\times \sqrt{9 M^{4}+16 \varepsilon M^{2}+16(1-\varepsilon)}\right] /[2(3+\varepsilon)] .
\end{aligned}
$$

Обратная зависимость $M\left(J_{p}\right)$ имеет вид

$$
M=\sqrt{\frac{(3+\varepsilon) J_{p}^{2}+2(1+3 \varepsilon) J_{p}-1+\varepsilon+4 \varepsilon^{2}}{(1+\varepsilon)\left(3 J_{p}+1+4 \varepsilon\right)}} .
$$

Условие изобаричности $\left(\tilde{N}_{1}=0\right)$, выполняемое вдоль границы струи, позволяет установить геометрическую кривизну скачка $A T$ :

$$
K_{\sigma}=-\sum_{j=1}^{4} A_{1 j} N_{j} / A_{15}
$$

и границы струи за ним $\left(\tilde{N}_{2} \equiv K_{\tau}\right)$ :

$$
K_{\tau}=C_{2} \sum_{j=1}^{4}\left(A_{2 j} A_{15}-A_{1 j} A_{25}\right) N_{j} / A_{15},
$$


а также градиент полного давления

$$
\tilde{N}_{3}=C_{3} \sum_{j=1}^{4}\left(A_{3 j} A_{15}-A_{1 j} A_{35}\right) N_{j} / A_{15}
$$

и с учетом (1) и других известных соотношений для скачков уплотнения - производные его интенсивности $(d J / d w)$ и других параметров течения (статического давления, энтропии, числа Маха) за скачком по направлению его падения, если известны параметры течения перед ним (в устье струи).

Дифференциальные условия динамической совместности успешно использовались ранее при анализе характеристик поля течения Прандтля-Майера [24], взаимодействия этих волн с догоняющими [20] и встречными [22] скачками уплотнения, а также позволили провести анализ плоского струйного истечения в окрестности кромки сопла [23] при условии задания всех характеристик потока перед сходящим с кромки скачком.

Широко известная [24-26] модель течения по расширяющемуся соплу с коническими образующими или по соплу с равномерным потоком в выходном сечении (модель сверхзвукового истечения из цилиндрического или сферического источника) предполагает следующие соотношения для основных неравномерностей перед скачком в точке $A[21,24]$ :

$$
N_{1}=-\frac{(1+\delta) \gamma M^{2} \sin \theta}{\left(M^{2}-1\right) r}, N_{2}=N_{3}=0, N_{4}=\delta / r
$$

где $r$ - полуширина или радиус выходного сечения сопла. При использовании этой модели безразмерные (умноженные на величину $r$ ) значения кривизн $\bar{K}_{\sigma}$ и $\bar{K}_{\tau}$, а также других дифференциальных характеристик скачка и течения в сжатом слое за ним, являются функциями четырех определяющих параметров $(M, J=1 / n, \gamma$ и $\theta)$.

Анализ дифференциальных характеристик поля течения перерасширенной газовой струи в окрестности кромки сопла производится далее на примере использования модели (9) во всем теоретически допустимом диапазоне определяющих параметров $\left(M>1,1<J \leq J_{m}\right.$, $\left.\gamma>1,0 \leq \theta \leq 90^{\circ}\right)$. Практическая реализация сверхзвукового безотрывного течения на выходе из сопла при каждом частном наборе значений $M, n, \theta$ и $\gamma$ во многом зависит от специфических особенностей технических устройств и процессов и в настоящей работе не рассматривается.

\section{1. Изменение геометрической кривизны скачка уплотнения, сходящего с кромки}

Согласно соотношению (6), кривизна $K_{\sigma}$ падающего скачка уплотнения, рассматриваемая как функция параметров истечения, претерпевает бесконечный разрыв в точке постоянного давления $\left(A_{15} \rightarrow 0\right.$ при $\left.J \rightarrow J_{p}\right)$.
Направление разрыва кривизны скачка, сходящего с кромки, при фиксированном значении $\gamma$ зависит от числа Маха истечения (в плоской струе), а также от угла раствора сопла (в осесимметричном случае).

Кривизна скачка, сходящего с кромки сопла в плоской струе, пропорциональна значению $\sin \theta / r$. Безразмерная кривизна $K_{\sigma}^{-}=r K_{\sigma} / \sin \theta$ при $J \rightarrow 1$ (вырождение скачка в слабый разрыв), $J=J_{m}$ (прямой скачок уплотнения) и $J=J_{\Gamma}$ определяется соотношениями

$$
\begin{gathered}
\lim _{J \rightarrow 1} K_{\sigma}^{-}=-\frac{(1-2 \varepsilon) M^{2}-2(1-\varepsilon)}{(1-\varepsilon) M\left(M^{2}-1\right)}, \\
\lim _{J \rightarrow J_{m}} K_{\sigma}^{-}=-\frac{M^{2}}{(1-\varepsilon)\left(M^{2}-1\right)}, \\
\lim _{J \rightarrow J_{\Gamma}} K_{\sigma}^{-}=-\frac{\left[(1-2 \varepsilon) M^{2}-2(1-\varepsilon)\right] \sqrt{M^{2}-1+\varepsilon}}{(1-\varepsilon) \sqrt{1+\varepsilon} M\left(M^{2}-1\right)} .
\end{gathered}
$$

Как отмечено в работе [23], существуют четыре диапазона чисел Маха с различной зависимостью кривизны скачка от нерасчетности плоской струи (см. кривые 1-4 на рис. 2,a). При малых числах Маха истечения (кривые 1) геометрическая кривизна падающего скачка положительна на всем практически важном промежутке $\left[1 ; J_{p}\right)$. Положительное значение кривизны означает, что падающий скачок $A T$ является выпуклым вниз в окрестности кромки (рис. $1, b)$, что, однако, не исключает его последующего перегиба.

При числе Маха $M_{a}=\sqrt{(2-\varepsilon) /(1-\varepsilon)}=1.483$ и интенсивности $J=1$ впервые возникает локальный минимум кривизны скачка, рассматриваемой как функция нерасчетности истечения (кривые 2 на рис. 2,a). Кривизна скачка уплотнения в точке локального минимума впервые становится равной нулю при $M_{b}=1.571$ и $J_{b}=1.242$ (кривые 3). При произвольном показателе адиабаты газа значения $M_{b}$ и $J_{b}-$ наибольшие вещественные корни уравнений

$$
\begin{gathered}
(3-4 \varepsilon) M_{b}^{6}-8\left(3-6 \varepsilon+4 \varepsilon^{2}\right) M_{b}^{4} \\
+32 \varepsilon(1-\varepsilon)\left(M_{b}^{2}+16(1-\varepsilon)^{2}=0, \quad \sum_{k=0}^{4} a_{k} J_{b}^{k}=0\right. \\
a_{4}=(1-\varepsilon)(3-4 \varepsilon)(3+5 \varepsilon) \\
a_{3}=-4(1-\varepsilon)\left(6+\varepsilon-3 \varepsilon^{2}+16 \varepsilon^{3}\right) \\
a_{2}=-2\left(7+36 \varepsilon-45 \varepsilon^{2}-94 \varepsilon^{3}+32 \varepsilon^{4}-32 \varepsilon^{5}\right), \\
a_{1}=4\left(4+11 \varepsilon+6 \varepsilon^{2}+39 \varepsilon^{3}+52 \varepsilon^{4}-16 \varepsilon^{5}\right), \\
a_{0}=13+62 \varepsilon+85 \varepsilon^{2}-16 \varepsilon^{4}+48 \varepsilon^{5}
\end{gathered}
$$

Дальнейшее увеличение числа Маха приводит к сдвигу левой точки нулевой кривизны до значения $J=1$ при $M_{c}=\sqrt{2(1-\varepsilon) /(1-2 \varepsilon)}=1.581$ (кривые 4). При умеренных и больших числах Маха кривизна скачка уплотнения, сходящего с кромки сопла, как правило, отрицательна, что соответствует его широко известной „бочкообразной“ форме, показанной на рис. 1, $a$. 

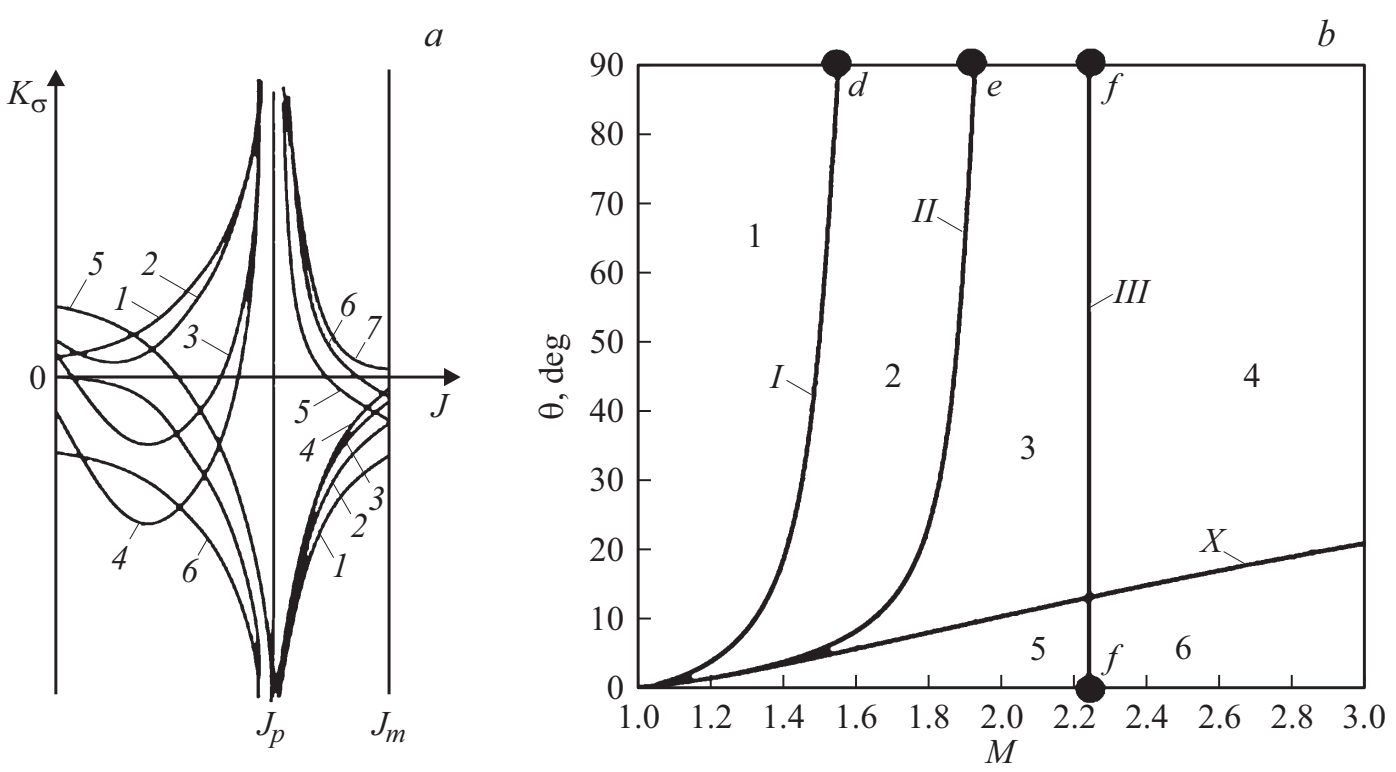

Рис. 2. Изменение кривизны скачка, сходящего с кромки, в зависимости от параметров истечения: $a-$ кривые $1-$ плоская струя при $M<M_{a}$ и осесимметричная струя в зоне $1 ; 2-$ плоская струя при $M<M_{a}$ и осесимметричная струя в зоне $2 ; 3-$ плоская струя при $M_{a}<M<M_{b}$ и осесимметричная струя в зоне $3 ; 4$ - плоская струя при $M_{b}<M<M_{c}$ и осесимметричная струя в зоне $4 ; 5-$ осесимметричная струя в зоне $5 ; 6-$ осесимметричная струя в зоне $6 ; 7-$ осесимметричная струя, $\theta=0$; $b$ - характерные области (зоны) параметров истечения осесимметричной струи.

Смена направления бесконечного разрыва кривизны скачка при $J \rightarrow J_{p}$ в плоском течении не наблюдается.

Геометрическая кривизна скачка уплотнения, сходящего с кромки в осесимметричной струе, зависит от параметров истечения более сложным образом. По этой причине изменение безразмерной кривизны $\bar{K}_{\sigma}=r K_{\sigma}$ в зависимости от нерасчетности осесимметричной струи рассматривается далее на плоскости параметров $(M, \theta)$, показанной на рис. $2, b$.

Изменение кривизны скачка в осесиммметричном течении при параметрах, соответствующих областям 1-4, аналогично приведенному на рис. 2, $a$ (кривые $1-4$ ) для плоской струи. Положительная и монотонно возрастающая на всем промежутке $\left[1 ; J_{p}\right)$ кривизна скачка в зоне 1 становится немонотонной в зоне 2 за кривой $I$. Кривая $I$ возникновения локального минимума кривизны скачка при $J=1$ описывается уравнением

$$
\operatorname{tg} \theta=-\frac{(1-\varepsilon)\left(M^{2}-1\right)^{3 / 2}}{3(1-\varepsilon) M^{4}-2(3-\varepsilon) M^{2}-(1-\varepsilon)}
$$

и оканчивается при $\theta=90^{\circ}$ и числе Маха

$$
M_{d}=\frac{3-\varepsilon+2 \sqrt{3-3 \varepsilon+\varepsilon^{2}}}{3(1-\varepsilon)}=1.551 .
$$

Кривая II появления скачков уплотнения с умеренной интенсивностью и отрицательной кривизной заканчивается при числе Маха $M_{e}=1.925$, вычисляемом как корень уравнения

$$
\sum_{k=0}^{4} b_{k} M_{f}^{2 k}=0, \quad b_{6}=-24 \varepsilon(2-3 \varepsilon)^{2},
$$

$$
\begin{gathered}
b_{5}=-80+672 \varepsilon-1036 \varepsilon^{2}+384 \varepsilon^{3}+252 \varepsilon^{4}, \\
b_{4}=296-930 \varepsilon+412 \varepsilon^{2}+886 \varepsilon^{3}-1096 \varepsilon^{4}+48 \varepsilon^{5}, \\
b_{3}=-(1-\varepsilon)\left(101+609 \varepsilon-1365 \varepsilon^{2}+1775 \varepsilon^{3}-192 \varepsilon^{4}\right), \\
b_{2}=-3\left(107-208 \varepsilon+423 \varepsilon^{2}-96 \varepsilon^{3}\right)(1-\varepsilon)^{2}, \\
b_{1}=\left(61+337 \varepsilon+192 \varepsilon^{2}\right)(1-\varepsilon)^{3}, \\
b_{0}=(1+48 \varepsilon)(1-\varepsilon)^{4} .
\end{gathered}
$$

Смена направления кривизны слабого (при $J \rightarrow 1$ ) скачка уплотнения в осесимметричном течении происходит при числе Маха $M_{f}=\sqrt{3(1-\varepsilon) /(1-3 \varepsilon)}=2.236$ и не зависит от полуугла $\theta$ раствора сопла (вертикальная линия III на рис. 2, $b$ ). Напомним, что в плоском случае аналогичное явление имеет место при $M_{c}=\sqrt{2(1-\varepsilon) /(1-2 \varepsilon)}=1.581$.

В отличие от плоской струи в осесимметричном течении возможна смена направления бесконечного разрыва кривизны скачка при $J \rightarrow J_{p}$. Параметры течения, при которых наблюдается смена направления разрыва кривизны, описываются уравнением

$$
\begin{gathered}
(1-\varepsilon)\left((3+\varepsilon) J_{p}+2+4 \varepsilon\right)\left(J_{p}-1\right)^{3 / 2} \\
\operatorname{tg} \theta=-\frac{\times \sqrt{\left(1+\varepsilon J_{p}\right)\left(3 J_{p}+1+4 \varepsilon\right)}}{\sqrt{J_{p}+\varepsilon}\left[(3+\varepsilon)(9 \varepsilon-1) J_{p}^{3}\right.} \\
\left.+\left(28+34 \varepsilon+82 \varepsilon^{2}+16 \varepsilon^{3}\right) J_{p}^{2}+c J_{p}+d\right] \\
c=33+94 \varepsilon+49 \varepsilon^{2}+48 \varepsilon^{3}, d=2(1+2 \varepsilon)(3+13 \varepsilon),
\end{gathered}
$$

где особые значения интенсивности $J_{p}(M)$ связаны с соответствующими числами Маха зависимостями (4), (5). 
Зависимость (10) показана на рис. $3, b$ кривой $X$, имеющей существующую при $\gamma>1.25$ горизонтальную асимптоту

$$
\theta=\operatorname{arctg} \frac{(1-\varepsilon) \sqrt{3 \varepsilon}}{9 \varepsilon-1}=49.684^{\circ}
$$

В области параметров $(M, \theta)$, расположенной ниже кривой $X$, находятся новые по сравнению со случаем плоской струи зоны 5 и 6 с монотонно убывающей на промежутке кривизной скачка (см. кривые 5 и 6 на рис. $2, a)$. При истечении равномерного осесимметричного потока $(\theta=0$, кривые 7 на рис. $2, a)$ отличие от смежных областей 5 и 6 состоит только в нулевых значениях кривизны скачка при $J \rightarrow 1$ и $J \rightarrow J_{m}$.

При малых значениях показателя адиабаты $(\gamma<1.25)$ области 5 и 6 с поведением кривизны скачка, нехарактерным для плоского истечения, расширяются до такой степени, что их верхняя граница (кривая $X$ ) оканчивается на линии $\theta=90^{\circ}$ при конечных числах Maxa.

Таким образом, при небольших полууглах раствора осесимметричного сопла влияние типа симметрии, как правило, является превалирующим, и характер изменения кривизны скачка не имеет аналогов в плоском течении.

\section{2. Завихренность струйного течения и изменение интенсивности падающего скачка}

Основная неравномерность $\tilde{N}_{3}$ потока за падающим скачком, согласно формуле Крокко для вихря, может служить мерой завихренности течения в сжатом слое. Анализ изменения завихренности $\tilde{N}_{3}$ в зависимости от параметров истечения основан на соотношении (8) и приведенном выше описании поведения кривизны $K_{\sigma}$ падающего скачка.

Характер изменения безразмерной завихренности $N_{3}^{-}=r \tilde{N}_{3} / \sin \theta$ в зависимости от нерасчетности плоской струи определяется числом Маха ее истечения. При $M<\sqrt{2}$ (кривые 1 на рис. 3, $a$ ) завихренность $N_{3}^{-}(J)$ отрицательна и монотонно убывает на всем промежутке $\left(1 ; J_{p}\right)$. Начиная с точки $J=1$ при $M=\sqrt{2}$, на графике функции $N_{3}^{-}(J)$ возникает положительный участок с точкой максимума (кривые 2). Положительность функции $N_{3}^{-}(J)$ соответствует традиционно предполагаемому усилению скачка уплотнения, падающего на плоскость или ось симметрии. Отрицательность значения $N_{3}^{-}$обычно проявляется при малых числах Маха в условиях парадокса фон Неймана и отсутствия решения для стационарных тройных конфигураций $[9,10,27,28]$. Местное ослабление скачков уплотнения, сходящих с кромки в этих условиях, может представлять интерес для анализа их дальнейшего падения и отражения [29].
Правая граница области усиления падающего скачка при $M>\sqrt{2}$ описывается соотношением (2) для огибающей $J_{\Gamma}(M)$ семейства ударных поляр и соответствует сверхзвуковому течению в сжатом слое за скачком.

Завихренность осесимметричного течения в сжатом слое описывается безразмерной функцией $\bar{N}_{3}=r \tilde{N}_{3}$. Как и безразмерная кривизна скачка, эта функция завихренности осесимметричного течения при больших углах раствора сопла изменяется во многом аналогично плоскому случаю, а при небольших углах $\theta-$ значительно отличается от него.

Описанная зависимость завихренности плоского течения от его нерасчетности характерна и для областей 1 и 2 параметров осесимметричной струи (рис. 3,b). Разделяющая эти зоны кривая $I$, соответствующая появлению слабых возмущений, усиливающихся в устье осесимметричной струи, характеризуется формулой

$$
\operatorname{tg} \theta=-\frac{(1-\varepsilon)\left(M^{2}-1\right)^{3 / 2}}{(1+3 \varepsilon) M^{4}+2(1-5 \varepsilon) M^{2}-7(1-\varepsilon)},
$$

и завершается при $\theta=90^{\circ}$ и числе Маха

$$
M_{g}=\sqrt{\left[2 \sqrt{2+\varepsilon+\varepsilon^{2}}-1+5 \varepsilon\right] /(1+3 \varepsilon)}=1.365 .
$$

Смена направления разрыва кривизны скачка на кривой $X$, описываемой условием (10), приводит к соответствующему изменению завихренности и других дифференциальных характеристик поля течения. В зонах 3 и 4 параметров осесимметричного течения, соответствующих небольшим углам раствора сопла, изменение завихренности сжатого слоя не имеет аналогов в плоской струе. Зона 3 характеризуется монотонным возрастанием функции $\bar{N}_{3}(J)$ на всем промежутке $\left[1 ; J_{p}\right)$, а зона 4 - появлением новых точек локального экстремума, которые возникают на кривой II из точек перегиба (см. также кривые 3 и 4 на рис. 3,a). Кривая II начинается при $M_{h}=1.809$ и $\theta=7.967^{\circ}$, где точка перегиба функции $\bar{N}_{3}(J)$ стремится к значению $J=J_{p}\left(M_{h}=3.338\right.$, и завершается при $M_{k}=4.849$ и $\theta_{k}=0$, где значение интенсивности скачка в точке перегиба $J_{k}=18.870$. Координаты точки $k$ определяются из соотношений $(5),(10)$ и нижеследующего:

$$
\begin{aligned}
& (1-\varepsilon)\left(J_{p}-1\right)^{3 / 2}\left(2+4 \varepsilon+(3+\varepsilon) J_{p}\right) \\
& \operatorname{tg} \theta=\frac{\times \sqrt{\left(1+\varepsilon J_{p}\right)\left(3 J_{p}+1+4 \varepsilon\right)}}{\left[(3+\varepsilon)(9 \varepsilon-1) J_{p}^{3}+\left(28+34 \varepsilon+82 \varepsilon^{2}+16 \varepsilon^{3}\right) J_{p}^{2}\right.} \text {. } \\
& \left.+\left(33+94 \varepsilon+49 \varepsilon^{2}+48 \varepsilon^{3}\right) J_{p}+d\right] \sqrt{J_{p}+\varepsilon}
\end{aligned}
$$

Изменение интенсивности $J$ скачка уплотнения характеризуется безразмерными функциями $W_{J}^{-}=$ $=r(d J / d w) / \sin \theta$ в плоском и $\bar{W}_{J}=r(d J / d w)$ в осесимметричном течении, где $w-$ направление падения скачка.

Отношение $I$ полных давлений потока на скачке уплотнения является монотонной функцией его интен- 

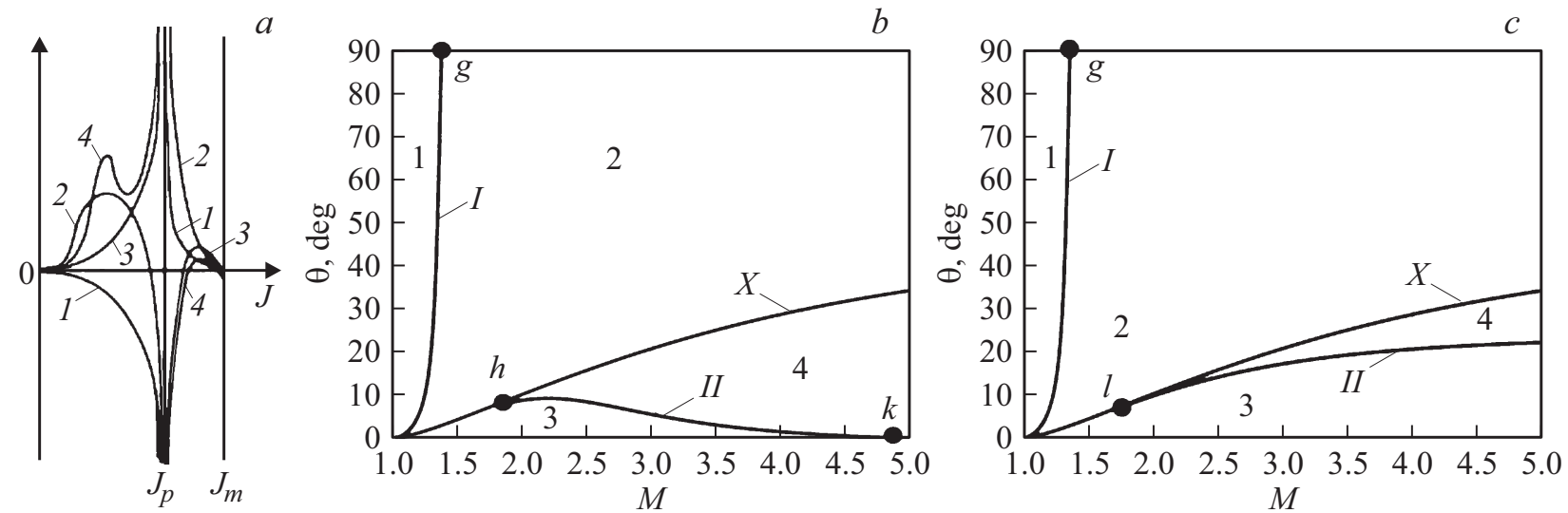

Рис. 3. Изменение завихренности $\left(N_{3}\right)$ потока в сжатом слое за падающим скачком и интенсивности скачка $(d J / d w): a-$ кривые 1 - плоская струя при $M<\sqrt{2}$ и осесимметричная струя в зоне $1 ; 2$ - плоская струя при $M>\sqrt{2}$ и осесимметричная струя в зоне $2 ; 3$ - осесимметричная струя в зоне $3 ; 4-$ осесимметричная струя в зоне 4 ; $b-$ области параметров с различным изменением величины $N_{3}$ в осесимметричной струе; $c$ - соответствующие области параметров с различным изменением интенсивности скачка.

сивности $J$ :

$$
I=\left(J \Omega^{\gamma}\right)^{-(1-\varepsilon) / 2 \varepsilon},
$$

где $\Omega=(1+\varepsilon J) /(J+\varepsilon)-$ отношение удельных объемов газа за скачком и перед ним согласно ударной адиабате Ренкина-Гюгонио. По этой причине изменение $d J / d w$ интенсивности скачка связано с функцией $\tilde{N}_{3}$ завихренности течения за ним

$$
\frac{d J}{d w}=\frac{2 J(J+\varepsilon)(1+\varepsilon J)^{2}}{(1-\varepsilon)(J-1)^{2} \sqrt{\left(J_{m}-J\right)(J+\varepsilon)+(1+\varepsilon J)^{2}}} \tilde{N}_{3} .
$$

Из (12), (13) очевидно, что знаки производных $W_{J}^{-}$ и $\bar{W}_{J}$ интенсивности скачка и функций завихренностей $N_{3}^{-}$и $\bar{N}_{3}$ в аналогичных условиях совпадают. В частности, в плоском течении поведение функции при и аналогично показаннному на рис. 3, $a$. Изменение интенсивности скачка в осесимметричном течении показано на рис. 3,a,c. При больших углах раствора сопла оно во многом соответствует плоскому случаю: убывание интенсивности скачка на всем промежутке $\left[1 ; J_{p}\right)$ в зоне 1 ; кривая $I$, описываемая уравнением (11) - граница между зонами 1 и 2; возможность роста интенсивности падающего скачка в зоне 2 ; смена направления разрыва функции $\bar{W}_{J}(J)$ при $J=J_{p}(M)$ на кривой $X$. В областях 3 и 4, где угол раствора сопла относительно мал, функция $\bar{W}_{J}(J)$ на всем промежутке $\left(1 ; J_{p}\right)$ положительна (кривые 3 и 4 на рис. $\left.4, a\right)$. Граница $I I I$ между зонами 3 и 4 начинается в точке $l$ $\left(M_{l}=1.776, \theta_{l}=7.582^{\circ}, J_{1}=J_{p}\left(M_{l}\right)=3.212\right)$ и имеет горизонтальную асимптоту.

Таким образом, при небольших углах раствора осесимметричного сопла падающий скачок усиливается, а завихренность течения за ним возрастает независимо от числа Маха истечения. Если же угол раствора достаточно велик, то, как и в плоской струе, при малых числах Маха наблюдается локальное падение интенсивности скачка и завихренности течения, а при умеренных и больших числах Маха - увеличение этих параметров.

\section{3. Статическое давление в сжатом слое}

Два основных фактора влияют на изменение статического давления за скачком при его сходе с кромки: уменьшение давления перед ним и изменение интенсивности скачка, рассмотренное в разд. 2. Если падающий скачок увеличивает свою интенсивность $(d L / d w>0)$, то эти факторы имеют противоположную направленность. При ослаблении сходящего с кромки скачка $(d L / d w<0)$ данные факторы сонаправленно приводят к уменьшению давления за скачком по мере его падения.

Изменение статического давления за скачком описывается безразмерными функциями $R_{w}^{-}=(r / \sin \theta) \times$ $\times(d \ln p / d w)$ и $\bar{P}_{w}=r(d \ln p / d w)$, вводимыми для плоского и осесимметричного истечения соответственно. Изменение функции $P_{w}^{-}(J)$ для плоской струи показано на рис. 4, $a$ (кривые $1-3$ ). Монотонное уменьшение функции $P_{w}^{-}(J)$ на промежутке $\left[1 ; J_{p}\right)$ (кривая 1 ) сменяется появлением локального максимума при числе Маха

$$
M_{r}=\sqrt{\left[\sqrt{9-4 \varepsilon+4 \varepsilon^{2}}-3+6 \varepsilon\right] / 4 \varepsilon}=1.166 .
$$

Единственное дальнейшее изменение вида функции $P_{w}^{-}(J)$ связано с появлением локального экстремума при числе Маха $M_{s}=\sqrt{(4-3 \varepsilon) /(1-3 \varepsilon)}=2.646$ на промежутке $\left(J_{p} ; J_{m}\right]$ глубокого перерасширения струи (кривая 3).

Размерное значение производной статического давления за слабым скачком описывается соотношением

$$
\lim _{J \rightarrow 1}\left(\frac{d \ln p}{d w}\right)=-\frac{(1+\delta) \gamma M \sin \theta}{r \sqrt{M^{2}-1}},
$$



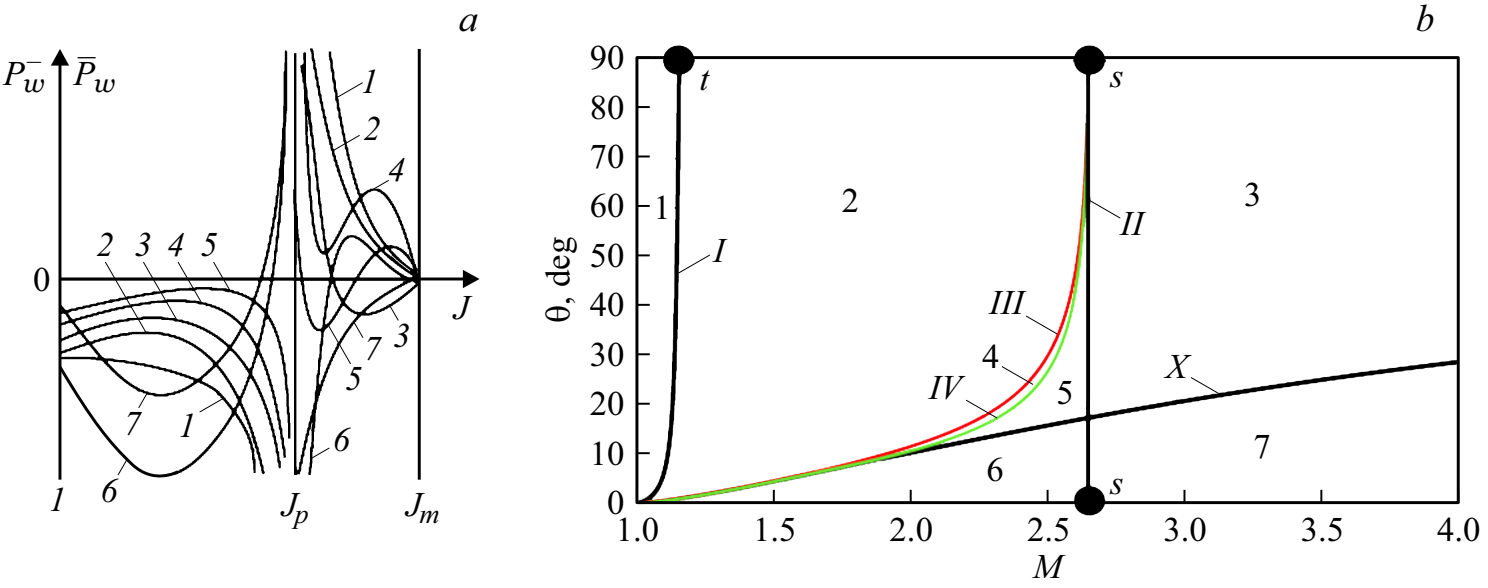

Рис. 4. Изменение статического давления в сжатом слое за скачком (функций $P_{w}^{-}=(r / \sin \theta)(d \ln p / d w)$ и $\bar{P}_{w}=r(d \ln p / d w)$ в плоской и осесимметричной струе соответственно): $a$ - кривые 1 - плоская струя при $M<M_{r}$ и осесимметричная струя в зоне 1 ; 2 - плоская струя при $M_{r}<M<M_{s}$ и осесимметричная струя в зоне $2 ; 3$ - плоская струя при $M>M_{s}$ и осесимметричная струя в зоне $3 ; 4$ - осесимметричная струя в зоне $4 ; 5$ - осесимметричная струя в зоне 5 ; 6 - осесимметричная струя в зоне 6 ; 7 - осесимметричная струя, зона $7 ; b-$ границы соответствующих зон для осесимметричной струи.

а при максимальной интенсивности скачка $\left(J \rightarrow J_{m}\right)$ соответствующая производная равна нулю как в плоском, так и в осесимметричном случае.

Изменение статического давления в сжатом слое осесимметричной струи рассматривается на плоскости $(M, \theta)$ параметров задачи (рис. $4, b)$. Поведение функции $\bar{P}_{w}(J)$ в зонах $1-3$, соответствующих большим углам раствора сопла, повторяет показанное кривыми $1-3$ на рис. 4, $a$ для различных случаев плоского истечения. Граница $I$ областей 1 и 2 возникновения немонотонности функции $\bar{P}_{w}(J)$ описывается соотношением

$$
\operatorname{tg} \theta=-\frac{(1-\varepsilon)\left(M^{2}-1\right)^{3 / 2}}{(1+3 \varepsilon) M^{4}+2(2-5 \varepsilon) M^{2}-7(1-\varepsilon)},
$$

и оканчивается при числе Маха

$$
M_{t}=\sqrt{\left[\sqrt{11-6 \varepsilon+4 \varepsilon^{2}}-2+5 \varepsilon\right] /(1+3 \varepsilon)}=1.158
$$

Кроме того, на плоскости $(M, \theta)$ присутствуют области 4 и 5 с двумя внутренними экстремумами функции $\bar{P}_{w}(J)$ на промежутке $\left(J_{p} ; L_{m}\right]$. Вертикальная граница II областей 5 и 3 , а также 6 и 7 с различным изменением функции $\bar{P}_{w}(J)$ на промежутке $\left(J_{p} ; J_{m}\right]$ соответствует тому же числу Маха $M_{s}=\sqrt{(4-3 \varepsilon) /(1-3 \varepsilon)}=2.646$, что и при плоском истечении.

Смена направления разрыва функции $\bar{P}_{w}(J)$ при $J \rightarrow J_{p}$ происходит на кривой $X$ и приводит к образованию областей 6 и 7. Внутри этих областей функция $\bar{P}_{w}(J)$ положительна на части промежутка $\left(1 ; J_{p}\right)$, а при $\theta=0$ - на всем этом промежутке. Следовательно, при небольших полууглах раствора сопла увеличение интенсивности скачка по мере его падения может превзойти расширение потока перед ним, и статическое давление за скачком увеличивается. Аналогичного увеличения статического давления за скачками умеренной интенсивности в плоском течении не наблюдается.

Помимо перечисленных характеристик поля течения перерасширенной или расчетной (при $J \rightarrow 1$ ) газовой струи, авторами проведен теоретический анализ ряда других параметров (таких, как определяемая соотношением (7) геометрическая кривизна границы струи или изменение $d M_{2} / d w$ (см. рис. 1) числа Маха $M_{2}$ в сжатом слое за скачком). Подтверждено существенное влияние вида симметрии струи на эти характеристики поля течения в сжатом слое, в особенности при небольших углах раствора сопла.

\section{Заключение}

Зависимости геометрических кривизн падающего скачка уплотнения и дифференциальных характеристик потока в сжатом слое за ним от параметров истечения в плоском и осесимметричном случаях качественно различаются. Влияние фактора осесимметричности особенно заметно при небольших значениях угла раствора сопла. Этот фактор позволяет реализовать слабо или умеренно перерасширенные осесимметричные течения со свойствами, не присущими аналогичным плоским струям (например, с положительной кривизной границы струи, что важно ввиду возможного развития неустойчивости Тейлора-Гертлера $[6-8])$.

Теоретические результаты, представленные в работе, совпадают с полученными численно при использовании метода характеристик второго порядка аппроксимации [30,31]. Они могут быть применены в дальнейшем как для анализа других струйных течений и развития соответствующих технологий, так и для верификации численных методов высокого порядка точности. 
Работа выполнена при финансовой поддержке Российского фонда фундаментальных исследований (проект 1608-01228).

\section{Список литературы}

[1] Zapryagaev V., Pickalov V., Kiselev N., Nepomnyashchiy A. // Theoret. Comp. Fluid. Dynamics. 2004. Vol. 18. N 2-4. P. 301-308.

[2] Киселев Н.П. Экспериментальное исследование структуры течения в слое смешения сверхзвуковой струи при наличии продольных вихрей. Автореф. дис. на соиск. уч. степ. канд. физ.-мат. наук. Новосибирск: ИТПМ им. С.А. Христиановича СО РАН, 2007. 17 с.

[3] Saric W.S. // Annual Review of Fluid Mechanics. 1994. Vol. 26. N 1. P. 379-409.

[4] Sescu A., Thompson D. // Theoret. Comp. Fluid. Dynamics. 2015. Vol. 29. N 1-2. P. 67-92.

[5] Lucas J.-M., Vermeersch O., Arnal D. // Europ. J. Mechan. B. Fluid. 2015. Vol. 50. P. 132-146.

[6] Терехова Н.М. // Доклады РАН. 1996. Т. 347. № 6. С. 759 762.

[7] Глазнев В.Н., Запрягаев В.И., Усков В.Н., Терехова Н.М., Ерофеев В.К., Григорьев В.В., Кожемякин А.О., Котенок B.A., Омельченко А.В. Струйные и нестационарные течения в газовой динамике. Новосибирск: Изд-во СО PAH, 2000. $200 \mathrm{c}$.

[8] Запрягаев В.И., Киселев Н.П., Павлов А.А. // Прикладная механика и техническая физика. 2004. Т. 55. № 3. С. 32 43.

[9] Васильев Е.И., Крайко А.Н. // Журнал вычислительной математики и математической физики. 1999. Т. 39. № 8. C. 1393-1404.

[10] Усков В.Н., Чернышов М.В. // Прикладная механика и техническая физика. 2006. Т. 47. № 4. С. 39-53.

[11] Горшков Г.Ф., Усков В.Н. // Прикладная механика и техническая физика. 2002. Т. 43. № 5. С. 49-54.

[12] Адрианов А.Л., Старых А.Л., Усков В.Н. Интерференция стационарных газодинамических разрывов. Новосибирск: Наука, 1995. $180 \mathrm{c}$.

[13] Uskov V.N., Mostovykh P.S. // Shock Waves. 2016. Vol. 26. N 6. P. 693-708.

[14] Brown W.F. // J. Mathematic. Physics. 1950. Vol. 29. N 4. P. 252-262.

[15] Eckert D. // Zeitschrift für Angewandte Mathematik und Mechanik (ZAMM). 1975. Vol. 55. N 6. P. 281-289.

[16] Emanuel G., Hekiri H. // Shock Waves. 2007. Vol. 17. N 1-2. P. 85-94.

[17] Mölder S. Curved shock theory // Shock Waves. 2016. Vol. 26. N 4. P. 337-353.

[18] Emanuel G. Shock Waves Dynamics: Derivatives and Related Topics. Boca Raton: CRC Press, 2012. 235 p.

[19] Smirnov N.N., Nikitin V.F., Alyari Shurekhdeli S. // J. Propulsion and Power. 2009. Vol. 25. N 3. P. 593-608.

[20] Silnikov M.V., Chernyshov M.V., Uskov V.N. // Acta Astronautica. 2014. Vol. 99. P. 175-183.

[21] Silnikov M.V., Chernyshov M.V. // Acta Astronautica. 2017. Vol. 135. P. $172-180$

[22] Мешков В.Р., Омельченко А.В., Усков В.Н. // Вестник СПбГУ. Сер. І. Математика. Механика. Астрономия. 2002. Вып. 2. С. 101-109.
[23] Silnikov M.V., Chernyshov M.V., Uskov V.N. // Acta Astronautica. 2014. Vol. 97. P. 38-41.

[24] Silnikov M.V., Chernyshov M.V. // Acta Astronautica. 2015. Vol. 109. P. 248-253.

[25] Дулов В.Г., Лукьянов Г.А. Газодинамика процессов истечения. Новосибирск: Наука, 1984. 234 с.

[26] Авдуевский В.С., Ащратов Э.А., Иванов А.В., Пирумов У.Г. Газодинамика сверхзвуковых неизобарических струй. М.: Машиностроение, 1989. 320 с.

[27] Гудерлей К.Г. Теория околозвуковых течений. М.: Изд-во ИЛ, 1960. $424 \mathrm{c.}$

[28] Gvozdeva L.G., Gavrenkov S.A., Silnikov M.V. // Acta Astronautica. 2015. Vol. 116. P. 36-42.

[29] Мостовых П.С., Усков В.Н. // Вестник СПбГУ. Сер. I. Математика. Механика. Астрономия. 2012. Вып. 1. С. $117-$ 127.

[30] Edward Ehlers F. // J. Society for Industrial and Applied Mathematics. 1959. Vol. 7. N 1. P. 85-100.

[31] Качкова О.Н., Наумова И.Н., Шмыглевский Ю.Д., Шулиншина Н.П. Опыт расчета плоских и осесимметричных сверхзвуковых течений газа методом характеристик. М.: ВЦ АН СССР, 1961. 60 с. 